\title{
An Overview of Aseptic Strategies Applied for In-house Polymerase Chain Reaction Techniques Discussed with Trouble Shootings
}

\author{
Kathiravan Vayanaperumal and Ranjith Kumar
}

\section{ABSTRACT}

Polymerase chain reaction which is the widely utilized method had made progressive imprint in scientific research has its solid focuses and inadequacies. It presents the chance of defilement through the spreading of amplicon mist concentrates in the laboratory environment. To help lessen the probability of tainting, good laboratory practice ought to be practiced consistently. This review is centers to setting up a PCR laboratory and techniques for counteraction and devastation of contamination, and quality control.

Keywords: Polymerase Chain Reaction, PCR Lab, Aseptic Technique, Quality Control.

Published Online: November 18, 2020

ISSN: $2684-5199$

DOI : 10.24018/ejbio.2020.1.6.119

Kathiravan Vayanaperumal*

Sankara Nethralaya Referral Lab,

Medical Research Foundation, Sankara Nethralaya, India.

(e-mail: kathiravan.vj@gmail.com)

Ranjith Kumar

Sankara Nethralaya Referral Lab, Medical Research Foundation, Sankara Nethralaya, India.

(e-mail: ranjiu99@gmail.com)

*Corresponding Author

\section{INTRODUCTION}

The polymerase chain reaction (PCR) is commonly used in molecular biology to make multiple copies of a specific DNA segment. It is must to develop various aseptic techniques and strategies in order to standardize and apply molecular biology methods of in-house polymerase chain reaction techniques in diagnostic or research laboratory. After its discovery, the experience with polymerase chain reaction pushed scientists to adapt multiple precautions and modification because, practically, polymerase chain reaction can fail for various reasons [1]. The sources of contaminants from samples carryover, reagents, disposable materials, and environment are huge and they can contribute to the contamination of the ultimate PCR outcome [2], [3]. This review aims to provide information on aseptic molecular laboratory practices employed to control troubleshooting in polymerase chain reaction techniques. The important technical issues that have been addressed are PCR laboratory design, workflow, assembling of materials and reagents, setting up reaction mixtures, pipetting techniques, aseptic cleaning and quality control.

\section{PCR LABORATORY DESIGN}

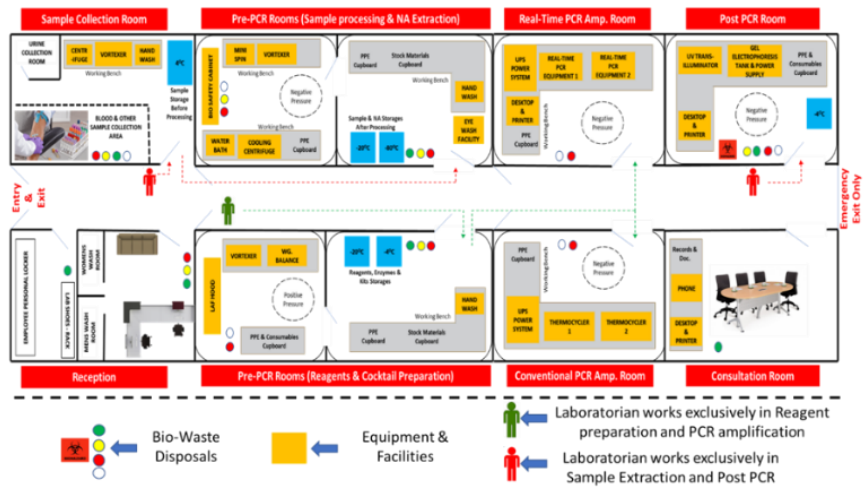

Fig. 1. PCR laboratory model.

In general, the PCR laboratory works include sample collection, sample processing, nucleic acid extraction, reaction mixture setting, amplification and post PCR analysis. Maintaining the laboratory in a contamination free environment is a highly challenging task because PCR is highly sensitive and the downside is that even trace amount of contaminating DNA may lead to amplification of nonspecific products [4]. A well-organized laboratory setup facilitates the prevention of contamination. The laboratory should be designated in such a way that at least three separate rooms should be allotted for various activities to take place. 
A separate pre-PCR clean room should be equipped with a laminar flow hood for PCR reagent and cocktail preparations and a bio safety cabinet for sample processing and nucleic acid extraction. Necessary equipment such as water bath, vortex mixer and mini centrifuge should be placed on the bench space provided in the room. Dedicated refrigerator and deep freezers for kit storage, reagents, enzymes and for storage of samples must made available.

A separate amplification room should be equipped only with real time PCR machines and thermal cyclers. This separate chamber reduces the possibilities of cross contamination with non-amplified materials from the environment or samples. Errors in annealing temperature, extension time or different cycling conditions gives number of troubleshoots such as no amplicon, low yield, multiple products and also stops the reaction working. Ensuring the program is correct on the thermal cycler and use the same thermal cycler for optimization and all future experiment because different cyclers can vary in ramping speed and temperature [5].

Post amplification procedures and analysis should be performed in a separate room. The Post-PCR room is where the gel electrophoresis equipment and the documentation system should be located. The post PCR room should be equipped with agarose gel disposal unit.

All the PCR rooms expect the clean room should be installed with the ventilation system that creates negative room pressure to allow air to flow into the isolation room but not escape from the room. Negative room pressure prevents cross-contaminations from room to room. [6] Corners of the walls should be rounded, makes easy to clean and it controls contamination [7].

\section{WORKFLOW}

The order of PCR laboratory works should be in one direction, i.e. the direction of workflow should start in prePCR and end in post-PCR [8]. Two laboratory personals must be employed to perform exclusive functions throughout the whole PCR process. The samples which contains DNA templates should be processed and nucleic acid extraction must be done only in pre-PCR room in a bio safety cabinet. Misusing this strategy will result in wrong size band amplification as a result of contamination. This can be cross checked by ensuring no band in negative control.

The other laminar air flow chamber available in pre-PCR room should be utilized only for setting reaction mixtures. The laboratory personal who is doing extraction should not be employed in settings. This is to avoid reagents being contaminated. Band in negative control is a result of aerosol contamination. Using fresh aliquot of reagents with proper pipetting techniques and regular change of gloves may minimize the error.

Amplified PCR tubes from the amplification room contain amplicons should never be introduced in the prePCR room. In nested or semi nested PCR techniques the first round amplified products is transferred to the secondround reaction mixtures. Amplicons from these products can easily contaminate future PCR or reagents, or equipment when it is transferred in pre-PCR room. So, the negative controls alone are transferred in pre-PCR, samples in amplification room and positive controls in post-PCR.

The laboratory personal who is doing specimen processing and nucleic acid extraction is only advised to add template to reaction mixture, handle positive controls and transfer of positive control products. Rest of the work is supported by another personal. These strategies shouldn't miss to follow and get diverted from unidirectional workflow. Care should be taken to regularly change personal protective equipment, specially gloves.

\section{ASSEMBLING MATERIALS AND REAGENTS}

Very important aspect of a PCR laboratory through which laboratory contamination can be minimized and to overcome troubleshoot is proper assembling of materials and equipment, storage of reagents, enzymes and kits, preparing master mix stocks, maintaining stock primers and diluted primers, etc.

Each room or hoods should have its own centrifuge, cyclomixers, pipettors, gloves, face masks, etc. Displacing pipettors between pre- and post-PCR or using extraction hood pipettors in setting hood will contaminate pipettors and result in band in negative control.

Reagents, enzymes, samples and mixtures used for PCR should be stored in dedicated refrigerators or deep freezers in order to maintain its stability. Stock kits, samples containing positive RNA viruses, extracted RNA were stored in $-80{ }^{\circ} \mathrm{C}$ deep freezer. RNA loses its stability and starts to degrade when not stored in between $-20{ }^{\circ} \mathrm{C}$ to $-80^{\circ} \mathrm{C}$. [9] In use kits, master mix stocks, diluted primers, positive samples, DNA should be stored in $-20^{\circ} \mathrm{C}$ [10].

Preparation of PCR master mix is done in a separately in clean room. Master mix is the mixture of dNTP, PCR buffer, ultra-pure water and taq-polymerase. With utmost sterility maintained and aseptic handling master mix stocks are prepared and stored separately away from samples at $-20^{\circ} \mathrm{C}$. It is advisable to use master mix stocks within ten days of preparation for better PCR sensitivity. Because dNTPs are very susceptible to freeze thawing and may get degraded and results in reaction stop working. Stock primers are diluted at short intervals and bulk preparation of primers are strictly avoided because frequent thawing and freezing will lose its stability [11].

\section{SETTING UP REACTION MIXTURE}

Setting up of reaction mixture is performed in pre-PCR room, where prior UV sterilized laminar air flow hood is used. Before setting up the reaction mixture the master mix and primers are brought to room temperature, make note that they should not be hand thawed. Labelling of vials are done and the reagents and mixtures are vortexed before aliquoting cocktail. Cocktail preparation is actually bulk preparation from which each individual reaction mixture is aliquoted. Change of gloves is recommended when reaction mixtures are aliquoted from cocktail. The reaction mixture consisting of DNA template, forward and reverse primers, taq polymerase, dNTPs and buffer containing magnesium 
ions are carried out in a $50 \mu \mathrm{l}$ reaction volume with $0.2 \mathrm{ml}$ thin wall polypropylene tubes. Improper handling and pipetting techniques will result in failure of polymerase chain reaction with lot of trouble shootings.

Trouble shootings such as absence of amplicon, low yield, nonspecific and wrong size band amplification may arise and the possible causes could be incorrect $\mathrm{MgCl}_{2}$ concentration, addition of insufficient or overabundance of primers, addition of wrong primers, and contaminations [12].

\section{PiPetting techniques}

One of the most efficient ways to prevent crosscontamination is through the use of a good pipetting technique. Accurate pipetting will improve the quality of results. Best pipetting practices includes correct pipette/tip combination, correct immersion depth, vertical pipette posture for liquid uptake, prewetting, correct liquid discharge and slow and continuous working pace [13]. Accurate volume should be aspirated and dispensed and splashing of samples or solutions should be avoided by careful opening and closing of caps each time.

The cap of the tube to which the solution to be dispensed is opened first and left in the rack. Followed by, the tube from which the solution to be aspirated in held in one hand and with the other hand the solution is pipetted from it and dispensed into the tube which is left in the rack. Use of sterile guarded tips will resist aerosol trapping them inside the barrier. Use different pipettes for pre- and post- PCR to prevent pipette contamination. Pipette contamination may result in band in the negative control.

\section{ASEPTIC CLEANING}

For extremely high-performance PCR laboratories proper aseptic cleaning should be carried out periodically before and after PCR work. Every work surface including bench space, pipettors, door handles, and hood should be wiped using $70 \%$ isopropanol and the materials to be discarded should be soaked in 1\% sodium hypochlorite solution [14]. Laminar airflow and biosafety cabinets were UV switched on overnight to sterilize the chambers and to switch on blower 15minutes before processing. Decontaminate the cabinet when use. Plan the work as needs be by utilizing separate clean and grimy sides inside the cabinet. Try not to obstruct the front or the rear grills of the biosafety cabinet. Discard wastes timely. It is advisable to use hand rubs at frequent intervals while working.

\section{QUALITY CONTROL}

With each amplification run appropriate positive and negative controls are included. A positive control assures proper performance of extraction and the negative control is used to check for the absence of contamination in the reagents, consumables, and environment. Trouble shootings such as no band in positive control or smearing appearance of band are the indications of low yield product. Nonoptimal annealing temperature and $\mathrm{MgCl}_{2}$ concentration, using direct cell lysate without extractions are the causes for the trouble shootings. Regularly, surface wipe test and settle plate testing should be performed to monitor the laboratory environment for contamination.

\section{SUMMARY}

The focus of this article was to provide a short review on aseptic method to eliminate contamination in PCR laboratory. Every PCR laboratory require its own needs and that should be carefully considered to operate this kind of laboratory successfully. Well-trained personnel, and automation are the two important aspects that will take molecular laboratories to next level.

\section{ACKNOWLEDGMENT}

We acknowledge the expert advice received from Dr HN Madhava, Director, Sankara Nethralaya Referral laboratory, Medical Research Foundation, Sankara Nethralaya, India, in the preparation of this article.

\section{REFERENCES}

[1] Kwok S, Higuchi R. Avoiding false positives with PCR. Nature 1989;339(6221):237. doi:10.1038/339237a0.

[2] Aslanzadeh J. Preventing PCR amplification carryover contamination in a clinical laboratory. Ann Clin Lab Sci. 2004;34(4):389-396.

[3] Varanat M, Maggi RG, Linder KE, Horton S, Breitschwerdt EB. Cross-contamination in the molecular detection of Bartonella from paraffin-embedded tissues. Vet Pathol. 2009;46(5):940-944. doi:10.1354/vp.08-VP-0259-B-BC.

[4] Kalle E, Kubista M, Rensing C. Multi-template polymerase chain reaction. Biomol Detect Quantif. 2014;2: 11-29. doi:10.1016/j.bdq.2014.11.002.

[5] Lorenz TC. Polymerase Chain Reaction: Basic Protocol Plus Troubleshooting and Optimization Strategies. J Vis Exp JoVE. 2012;(63). doi:10.3791/3998.

[6] Guidelines for Environmental Infection Control in Health-Care Facilities: (545922006-001). Published online 2003. doi:10.1037/e545922006-001.

[7] Bio-Pharmaceutical Cleanroom Design Guidelines. Accessed June 10, 2019. https://www.portafab.com/bio-pharmaceutical-cleanroomdesign.html.

[8] Mifflin T. Setting up a PCR laboratory. CSH Protoc. 2007; 2007: pdb. top14. doi:10.1101/pdb.top14.

[9] Fabre A-L, Colotte M, Luis A, Tuffet S, Bonnet J. An efficient method for long-term room temperature storage of RNA. Eur J Hum Genet. 2014;22(3):379-385. doi:10.1038/ejhg.2013.145.

[10] January 7 P, Share 2014. Free Up Your Freezer with These RoomTemperature DNA Storage Solutions. Accessed June 11, 2019. http://www.biocompare.com/Editorial-Articles/152844-Free-UpYour-Freezer-with-These-Room-Temperature-DNA-StorageSolutions/.

[11] 7 Tips to Make PCR Primers Last Longer and PCR Reactions Run Better. GEN - Genetic Engineering and Biotechnology News Published June 27, 2012. Accessed June 11, 2019 https://www.genengnews.com/magazine/7-tips-to-make-pcr-primerslast-longer-and-pcr-reactions-run-better/.

[12] PCR Troubleshooting | LSR | Bio-Rad. Accessed June 11, 2019. http://www.bio-rad.com/en-us/applications-technologies/pcrtroubleshooting?ID=LUSO3HC4S

[13] Pushparaj PN. Revisiting the Micropipetting Techniques in Biomedical Sciences: A Fundamental Prerequisite in Good Laboratory Practice. Bioinformation. 2020;16(1):8-12. doi:10.6026/97320630016008.

[14] Standard_Operating_Procedures.pdf. Accessed June 11, 2019 https://www.icmr.nic.in/sites/default/files/guidelines/Standard_Operat ing_Procedures.pdf. 


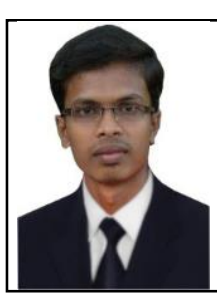

Kathiravan Vayanaperumal from Thoothukudi, Tamilnadu, India. Done his Master degree in Medical Laboratory Technology at Loyola College, Chennai. Currently, works as Deputy Technical Manager at Sankara Nethralaya Referral Laboratory, Medical Research Foundation, Chennai. Experience in molecular microbiology diagnostic techniques pertaining to ocular microbiology. Interested to study and find research gaps in the field of clinical virology.

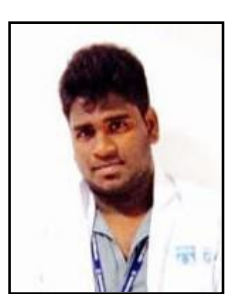

Ranjith Kumar from Chennai, Tamilnadu, India. Got his first degree from The Tamil Nadu Dr. M.G.R. Medical University, Chennai. Working as Senior Laboratorian at Sankara Nethralaya Referral Laboratory, Medical Research Foundation, Chennai. Experience in Microbiology, Serology and Mycobacteriology. 